The University of Stockholm has received from Mrs. Amanda Ruben the sum of 50,000 kroner to found a readership in experimental zoology.

DR. B. C. CRowell, professor of pathology and bacteriology, University of the Philippines, has been appointed director of the Graduate School of Tropical Medicine and Public Health of that university. This school gives courses which in one year lead to the degree of Doctor of Tropical Medicine and in two years to Doctor of Public Health.

Dr. H. B. FanthaM, of Christ's College, Cambridge, has been appointed to the professorship of zoology at the South African School of Mines and Technology, Johannesburg, and Dr. C. E. Moss, of Emmanuel College, has been appointed professor of botany in the same institution.

We learn from Nature that Dr. Johanna Westerdijk has been appointed associate professor of phytopathology in the University of Utrecht. She is said to be the first woman to receive such an appointment in Holland.

\section{DISCUSSION AND CORRESPONDENCE} THE LIMIT OF THE SPECTRUM IN THE ULTRAVIOLET

IN the Astrophysical Journal for March, 1916, I gave an account of my work in the extreme ultra-violet. During the past year I have continued my investigations in the same field; the results have not been commensurate with the labor, but it is perhaps worth while to make a brief report of them.

I have not changed the general design of my spectroscope but I have replaced the $100 \mathrm{~cm}$. grating by one of $50 \mathrm{~cm}$. radius, thus halving the light path and considerably reducing the volume to be exhausted. My source of light is still a quartz discharge tube, but I have so altered the design that the end of the capillary can be brought much nearer the slit of the spectroscope than before; I have considerably increased the potential of the transformer; as before, I employ helium at one or two millimeters pressure to fill my spectroscope and discharge tube.
The net result of these changes is that I have certainly extended the spectrum from 600 to the neighborhood of 510 Ångströms; a trace of a line exists on my very best negative near 450 Ångströms, but it is far too faint to afford trustworthy evidence.

From time to time during the past five or six years I have tried Wood's miniature arc in vacuum, and a variety of vacuum spark arrangements, recently I have repeated the more promising of these experiments. None of these sources appear to yield lines in the most refrangible region. Helium continues the most promising source: THEODORE - LYMAN

$$
\begin{aligned}
& \text { J'tifterson Physical Laboratory, } \\
& \text { HARVARD UNIVERSITY, CAMBRIDGE, } \\
& \text { February 14, } 1917
\end{aligned}
$$

\section{THE FOUNDATIONS OF DYNAMICS AND DADOURIAN'S ANALYTICAL MECHANICS}

My attention was called recently to a review of the second edition of my "Analytical Mechanics" by Professor E. W. Rettger, which appeared in ScIEnce (No. 1130) last summer when I was in the mountains and did not see it. The review on the whole was favorable and would not have tempted the author of the book to make an answer at this late date were it not for the fact that the two questions raised by the reviewer bear upon the foundations of the science of mechanics.

The first of these is directed against my direct application of the laws of vectors to the directed magnitudes of mechanics:

Before we apply the law of vector addition to any kind of quantity, ought we not first assure ourselves that the parallelogram law holds for these quantities? Since force, for instance, is a directed quantity (italics are mine) does it follow that the parallelogram law holds for forces?

I would answer both of these questions in the affirmative. We have no right to apply vector operations to "any kind" of quantity. We ought to assure ourselves that the quantity in question is a "directed quantity" before treating it as such. But having once assured ourselves of this fact we need not hesitate to apply to it the parallelogram law or any other law of directed quantities. 
Vector algebra is the science of directed lines, or displacements, in space as ordinary algebra is the science of numbers. We do not hesitate to apply the laws of ordinary algebra to quantities which can be represented by numbers, we need no more have any compunction about applying the laws of vectors to quantities which can be represented by directed lines. The expression "the law of parallelogram of forces" is a provincialism for which there is about as little justification as there would be for a "law of the addition of apples" in arithmetic. The addition law of arithmetic is a law of numbers and is not peculiar to apples; it can be applied to apples not because they have certain desirable properties, but because they can be counted. Similarly, the parallelogram law is a law of displacements or directed lines, and not at all characteristic of forces, but it can be applied to forces because these have among other physical properties those of direction and of magnitude and consequently may be represented by directed lines.

This is the precise point of view which I have adopted in my book toward the directed magnitudes of mechanics. After giving a clear and concise exposition of the laws of addition and resolution of vectors in the first chapter I have applied them to directed quantities without hesitation. This mode of procedure is not only correct, but it is straightforward and simple, as the reviewer admits when he says:

If the author is correct... then certainly the theory underlying the composition and resolution of directed quantities becomes very simple.

The second question which Professor Rettger raises has to do with my formulation of the principle underlying the science of dynamics. In my book I have based dynamics upon the following principle, which I have called the action principle:

The vector sum of all the external actions to which a system of particles or any part of it is subject at any instant vanishes.

$$
\Sigma \mathbf{A}=\mathbf{0} \text {. }
$$

A particle may be acted upon by other particles and by the ether. The action of one particle upon another particle is known as a force. The action of the ether upon a particle I have called a kinetic reaction. Therefore the action principle states

$$
\Sigma(\boldsymbol{F}+\mathbf{q})=0,
$$

where $\mathbf{F}$ denotes a force and $\mathbf{q}$ a kinetic reaction. The kinetic reaction on a particle is oppositely directed from and proportional to the acceleration and the constant of proportionality is the characteristic constant of the particle known as mass. Therefore we have

$$
\boldsymbol{\Sigma}(\mathbf{F}-m a)=0 \text {. }
$$

Commenting upon this principle, Professor Rettger says :

The reviewer does not wish to say that the author is wrong in his conception. All he wishes to say is that he entirely fails to appreciate the author's point of view.

This lack of appreciation is due, it seems to me, to a lack of clear understanding, indicated by the following questions, of the nature and function of the kinetic reaction.

Why is it that the ether acts on a body only when it is being accelerated and not when the body is moving with constant velocity?

If kinetic reaction is the action of the ether on a particle, and if it is the same kind of a quantity as force (is a force in fact), and if the resultant force $F$ acting on a particle and the kinetic reaction $q$ are always equal in magnitude but opposite in direction (both equal to ma in magnitude), why is the body not in equilibrium?

If in the first of these questions the term "why" is used in the metaphysical sense, there is no answer for it, except possibly the equally metaphysical answer "because." On the other hand, if it is used to mean "how is this fact correlated with other facts?" I would state that the answer belongs to electrodynamics and not to mechanics and would refer the reviewer to a modern treatise on electrodynamics, Lorentz's book on "Electron Theory," for instance, where the question is answered at length.

Answering the second question, one might state: "The body is not in equilibrium for the same reason that a particle revolving in a circle is not in static equilibrium in spite of 
the fact that the so-called 'centrifugal' and 'centripetal' forces acting upon the particle are equal and oppositely directed." I am afraid the reviewer has overlooked the fact that a particle is in static equilibrium when and only when the sum of the forces due to other material bodies acting upon the particle equals zero. When this condition is not satisfied the particle is accelerated and by virtue of the acceleration the kinetic reaction comes into play. This kinetic reaction is equal and opposite to the resultant of the forces due to the material bodies. If it were not for the kinetic reaction a finite force would have given a body an infinite velocity in a finite time.

The kinetic reaction is of the same nature as a force and might be called a force, but that would tend to confound the cause with the effect. It would further necessitate changing the statement of the conditions of equilibrium as well as of motion. It was in order to keep the old concept of force as an action which causes acceleration and to distinguish between cause and effect that I refrained from applying the term force to kinetic reactions.

The concept of kinetic reaction is not new. It has been known to other authors of textbooks of mechanic as centrifugal force, inertia force, or inertia reaction. The thing that is new about kinetic reaction in my book is the full recognition it receives and the clear cut treatment which differentiates it from accelerating forces. I have preferred the name kinetic reaction to inertia reaction because it is just as much an acceleration-reaction as an inertia-reaction.

I claim that the point of view which I have adopted in my book has important philosophical and pedagogical advantages over the common point of view. The former has enabled me to differentiate between purely geometrical laws and dynamical principles, between kinematical relations and dynamical equations, between what is fundamental and what is derived in mechanics. I have postulated a single dynamical principle which is not only simple and sound, but is correlated with the equally fundamental principles of electrodynamics. Upon this single principle
I have based the entire subject, deriving from it all the other dynamical laws and principles used in elementary mechanics, such as Newton's three laws of motion, the principles of the conservation of energy, of linear momentum and of angular momentum.

Before closing this communication I would like to call the attention of teachers of mechanics to the following principle which I have introduced in the second edition of my book and have called it the angular action principle.

The vector sum of all the external angular action to which a system of particles or any part of it is subject at any instant vanishes:

or

$$
\Sigma \mathbf{A}_{a}=0 \text {, }
$$

$$
\Sigma\left(\mathbf{G}+\mathbf{q}_{a}\right)=0,
$$

where $\mathbf{G}$ denotes the moment of force about a given axis and $\mathbf{q}_{a}$ denotes the moment of the kinetic reaction of a particle about the same axis, the latter I have called the angular kinetic reaction. This principle, which is directly applicable to rotating systems, is equivalent to and derived from the action principle.

It can be easily shown from the angular action principle that the torque equation

$$
G=I \frac{d \omega}{d t}
$$

holds good only when the center of mass of the moving system remains at a constant distance from the axis of rotation, a point which has eluded most authors of textbooks of mechanics.

In conclusion I would state that the two action principles are simple statements of the following two sets of equations used in general dynamics.

$$
\begin{aligned}
& \Sigma(X-m \ddot{x})=0, \\
& \Sigma(Y-m \ddot{y})=0, \\
& \Sigma(Z-m \ddot{z})=0, \\
& \Sigma[y(Z-m \ddot{z})-z(Y-m \ddot{y})]=0, \\
& \Sigma[z(X-m \ddot{x})-x(Z-m \ddot{z})]=0, \\
& \Sigma[x(Y-m \ddot{y})-y(X-m \ddot{x})]=0 .
\end{aligned}
$$

H. M. Dadourian

YALE UNIVERSITY

THE SYNCHRONIC BEHAVIOR OF PHALANGIDE

Professor H. H. Newman's note in a recent number of SoIEnce reminds me that in $1901 \mathrm{I}$ 